\title{
Frequency of QTc Interval Prolongation In Pediatric and Young Adult Patients on Methadone for Cancer Related Pain
}

\author{
Kevin Madden MD; Eunji Jo MS; Janet Williams, MBA, MPH; Diane Liu MS, Eduardo Bruera, MD \\ MD Anderson Cancer Center, Houston, TX, USA
}

\section{Background:}

- Methadone has been reported to prolong the corrected QT (QTC) interval and increase the risk of torsades de pointes

- Most studies examining QTc interval prolongation in children are retrospective, limiting conclusions

\section{Methods:}

- All patients followed a standardized protocol

- Electrocardiograms (ECGs) were obtained at:

- Baseline (day starting methadone to 14 days prior), 1-2 weeks, and 4-6 weeks later

- QTc values were manually calculated using the Bazett formula. QTc prolongation was defined as:

- $\geq 460$ milliseconds (ms) for pre-pubertal children

$-\geq 470$ ms for pubertal males

$-\geq 480 \mathrm{~ms}$ for pubertal females

\section{Results:}

- Baseline: ECGs were completed in 42 patients

- No patients had QTc prolongation at baseline

- 1-2 weeks: 38/42 (91\%) had follow up ECGs

- $1 / 38(3 \%)$ patient had QTc prolongation at 1-2 weeks

- The patient had a history of prolonged QTc that the family did not initially report

- 4-6 weeks: $31 / 42$ (74\%) had follow up ECGs

- No patients had QTc prolongation at weeks 4-6

- No patients had torsades de pointes, ventricular fibrillation, or sudden death

- Compared to baseline QTc, the QTc interval at 1-2 weeks $(P=.05)$ and 4-6 weeks $(P=.01)$ were significantly longer but not clinically relevant

- Age greater than 18 years of age $(P=.11)$, gender $(P=.84)$ and total methadone daily dose $(P=.06)$ were not associated with an increased QTc interval

\section{Conclusions:}

- Methadone is a safe medication to use in children and young adults with cancer-related pain

- Clinically significant prolongation of the QTc interval occurred only in one patient, who had a history of prolonged QTC

- Prolonged QTc is rare in this population

- Dose of methadone is not associated with QTc value

- If the baseline ECG is normal, and there are no other risk factors follow-up ECGs may not be warranted

\section{Table 1. Baseline characteristics of patients}

\begin{tabular}{lc}
\hline & $\mathrm{n}=42(\%)$ \\
\hline Median age (range) & $16(1-25)$ \\
Female & $18(43 \%)$ \\
White & \\
Hispanic & $27(64 \%)$ \\
Asian & $8(19 \%)$ \\
African American & $2(5 \%)$ \\
Others/unknown & $2(5 \%)$ \\
Primary Oncologic Diagnosis & $3(7 \%)$ \\
Sarcoma & \\
Leukemia/Lymphoma & $20(48 \%)$ \\
Central Nervous System Tumor & $15(36 \%)$ \\
Neuroblastoma & $3(7 \%)$ \\
Laryngeal cancer & $2(5 \%)$ \\
Ovarian cancer & $1(2 \%)$ \\
\hline
\end{tabular}

\begin{tabular}{|c|c|c|c|c|c|}
\hline & $\begin{array}{c}\text { Baseline } \\
(\mathrm{n}=42)\end{array}$ & $\begin{array}{c}1-2 \text { weeks } \\
(n=38)\end{array}$ & $P$-value ${ }^{a}$ & $\begin{array}{c}4-6 \text { weeks } \\
(n=31)\end{array}$ & $P$-value ${ }^{\mathrm{b}}$ \\
\hline Median QTc, ms (IQR) & $391(377,400)$ & $399(374,411)$ & .05 & $393(379,423)$ & .01 \\
\hline QTc $\geq$ upper limit of normalc & $0(0 \%)$ & $1(2.6 \%)$ & & $0(0 \%)$ & \\
\hline Median methadone dose, mg (IQR) & - & $7.5(5,10)$ & & $5(4.4,10)$ & \\
\hline $\begin{array}{l}\text { Median methadone dose, } \\
\mathrm{mg} / \mathrm{kg} / \text { dose (Min, Max) }\end{array}$ & & $0.1(0.05,0.21)$ & & $0.1(0.05,0.32)$ & \\
\hline
\end{tabular}

C Upper limit of normal for: pre-pubertal children $=460 \mathrm{~ms}$; pubertal males $=470 \mathrm{~ms}$; pubertal females $=480 \mathrm{~ms}$

\begin{tabular}{|c|c|c|c|}
\hline & $\begin{array}{c}1-2 \text { weeks } \\
\mathrm{n}=38\end{array}$ & $\begin{array}{c}\text { 4-6 weeks } \\
\mathrm{n}=31\end{array}$ & $P$-value \\
\hline \multicolumn{4}{|c|}{$\begin{array}{l}\text { Number (\%) of patients on medications } \\
\text { that: }\end{array}$} \\
\hline Directly increase QTc & $33(87 \%)$ & $25(81 \%)$ & \\
\hline Indirectly increase QTC & $23(61 \%)$ & $16(52 \%)$ & \\
\hline \multicolumn{4}{|c|}{$\begin{array}{l}\text { Number [median, (IQR)] of medications } \\
\text { that: }\end{array}$} \\
\hline $\begin{array}{l}\text { Directly increase QTc } \\
\text { Association with QTc }\end{array}$ & $1(1,2)$ & $1(1,2)$ & .15 \\
\hline $\begin{array}{l}\text { Indirectly increase QTC } \\
\text { Association with QTc }\end{array}$ & $1(0,1)$ & $0(0,1)$ & \\
\hline Serum electrolyte levels, medi & & & \\
\hline Calcium $(\mathrm{mg} / \mathrm{dL})$ & $8.9(8.6,9.4)$ & $9.6(8.6,9.8)$ & \\
\hline $\begin{array}{l}\text { Association with QTC } \\
\text { Magnesium (ma/dL) }\end{array}$ & $18(1719)$ & $19(182 ?)$ & 17 \\
\hline Association with QTC & (1.0) & $1.0(1.0,2 . c)$ & .53 \\
\hline $\begin{array}{l}\text { Potassium (mEq/L) } \\
\quad \text { Association with QTc }\end{array}$ & $4.0(3.7,4.3)$ & $4.1(3.9,4.3)$ & $.02^{\mathrm{a}}$ \\
\hline
\end{tabular}

a One unit decrease of potassium ( $\mathrm{mEq} / \mathrm{L}$ ) was significantly correlated with a $17 \mathrm{msec}$ increase in the QTC

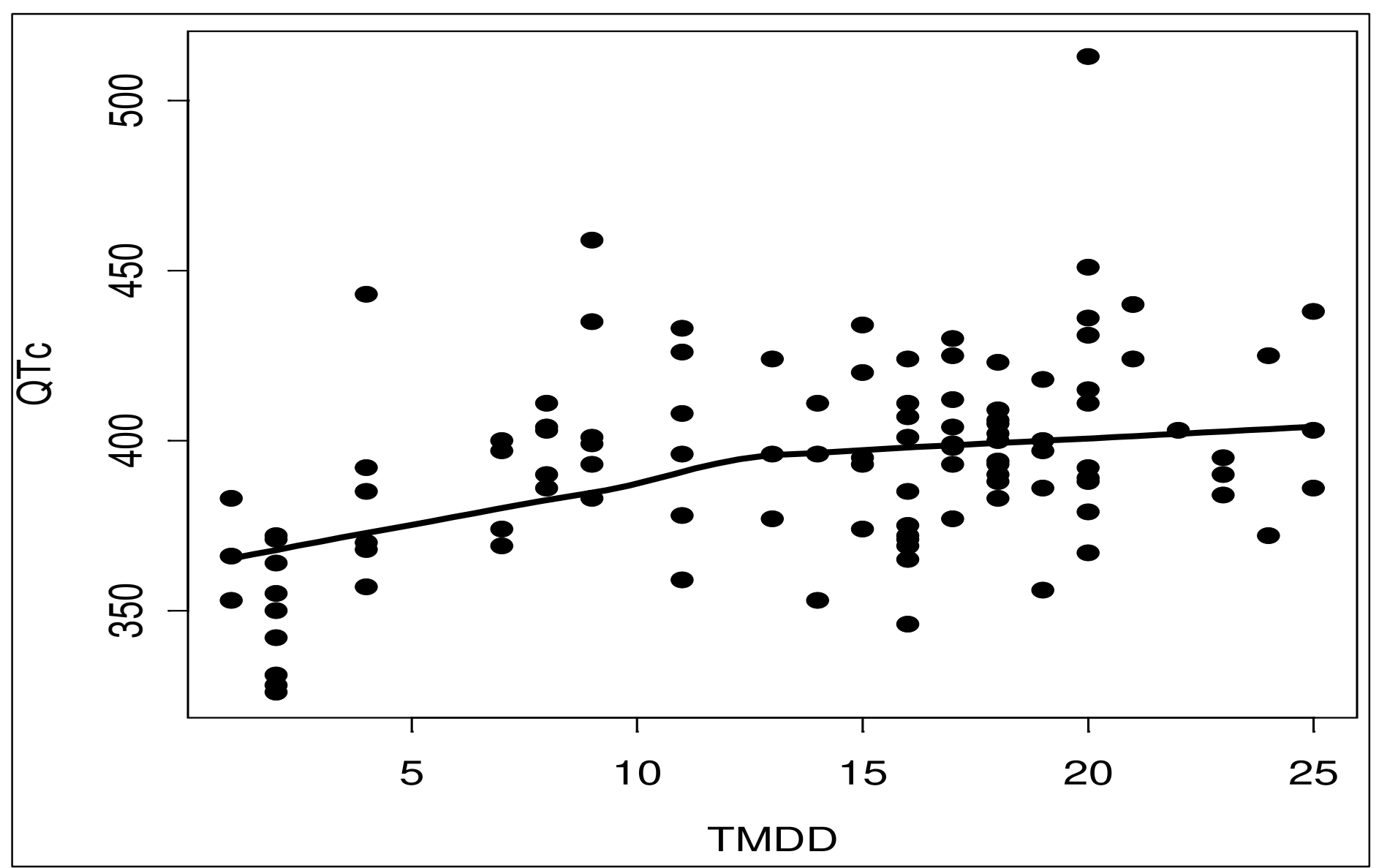

